\title{
Energy Efficient Virtual Machine Placement in Data Center
}

\author{
Ashwini S. Bhujbal \\ Zeal College of \\ Engineering, Pune
}

\author{
Pranoti C. Bansode \\ Zeal College of \\ Engineering, Pune
}

\author{
Pallavi P. Dukale \\ Zeal College of \\ Engineering, Pune
}

\author{
Sayali S. Gaikwad \\ Zeal College of \\ Engineering, Pune
}

\author{
N. R. Shikalgar \\ Assistant Professor, \\ Zeal College of Engineering, Pune
}

\begin{abstract}
The rising trend of e-commerce and cloud computing are just two reasons for the growing demand for Data Centers (DCs). DCs are among the largest global energy consumers in relation to the total global energy consumption. The rising numbers of DCs are an increasingly negative impact on the environment. This may be caused by the DCs themselves as well as by the power generation that is needed by the DCs. Thus, the utilization of servers in most data centers can be improved by adding virtualization and selecting the most suitable host for each Virtual Machine (VM). The problem of VM placement is an optimization problem to achieve multiple goals. To overcome those problems we design the allocator in order to accept as many VM requests as possible, taking into account the power consumption of the network devices. It can be covered through various approaches such as allocator policy (Best Fit/Worst Fit), allocation strategy (Single/Multiobjective optimization), and network resources. Each approach aims to simultaneously reduce power consumption, maximize resource utilization and avoid traffic congestion.
\end{abstract}

\section{Keywords}

Virtual Machine[VM], Data Center [DC], IT Resource Allocators [ITRA], First Fit[FT], Best Fit[BF], Worst Fit[WF], Multi-Objective Dynamic Allocator[MODA], Fuzzy Logic Controller[FLC], Analytic IT Resource Allocators[A-ITRA], Fuzzy IT Resource Allocators[FITRA].

\section{INTRODUCTION}

Cloud computing is a technology that enables computing resources, software, or data to deliver on demand services over the Internet. These resources have become cheaper, more powerful and more ubiquitously available than ever before. The cloud computing stack consists of three types of cloud service models: Infrastructure, Platform and Software, which are built upon the Hardware layer. Since provisioning Virtual Machines (VMs) is fundamental to provide infrastructure services, one can say that virtualization is the key concept of cloud computing. According to Mike Adams, director of product marketing at VMware1, "Virtualization software makes it possible to run multiple operating systems and multiple applications on the same server at the same time. It enables businesses to reduce IT costs while increasing the efficiency, utilization and flexibility of their existing computer hardware." However, VMs need to be adequately placed to fulfill performance goals, to optimize network flows, and to reduce CPU, storage and energy costs. VM placement optimization processes may be traffic-aware, energy-aware, application-aware, network topology-aware, data-aware, or a combination of these.
In recent years, the problem of allocating VMs to suitable Physical Machines (PMs) has been studied for efficiency and quality purposes. On the provider side, these solutions map VMs to PMs to optimize server efficiency, allowing some servers to hibernate or shut down depending on load conditions. On the consumer side, these solutions maximize Quality of Service (QoS) and Quality of Experience (QoE).

\section{LITERATURE SURVEY}

2.1 Green Power Management with Dynamic Resource Allocation for Cloud Virtual Machines

With the development of electronics in governments and business, the implementations of these services are increasing demand for servers. Continued expansion of servers represents our need for more space, power, air conditioning, network, human resources and other infrastructure. Regardless of how powerful servers now become, we do not make good use of all resources and strive for the waste.

Our approach is to overcome problem of load balancing for virtual machine management on cloud. It includes three main phrases:

(1) Supporting green power mechanism,

(2) Implementing virtual machine resource monitor onto Ganglia Software. [1].

2.2 Using the Multiple Knapsack Problem to Model the Problem of Virtual Machine Allocation in Cloud Computing:

Cloud computing is a new computing paradigm which has the provision of computational resources like services accessed over the internet. In this paradigm, computing resources are pooled and allocated according to customer demand. The growing demand for this new type of service has led to increased use of energy on the part of service providers, due to the need to maintain the computing infrastructure, becoming one of the leading providers of cost factors.

In this context, solutions have been trying whatever possible to meet the customer's requirements for resources consuming minimum power required. Thus, this work consists of an improvement, using the modeling of the multiple knapsack problems, with a mechanism for allocating resources Allocator addresses the issue of energy saving. Furthermore, a comparative analysis of the proposed solution with the original mechanism to evaluate the performance modification is made. Another improvement would be to use Ganglia software a formula defining how good the allocation of a VM is on a server regarding energy consumption and power balancing on the servers together. 
2.3 Multi-objective virtual machine placement in virtualized data centre environments:

Server consolidation using virtualization technology has become increasingly important for improving data center efficiency. It enables one physical server to host multiple independent virtual machines (VMs), and the transparent movement of workloads from one server to another. Finegrained virtual machine resource allocation and reallocation are possible in order to meet the performance targets of applications running on virtual machines. On the other hand, these capabilities create demands on system management, especially for large-scale data centers.

Our approach is to manage the mappings of workloads to VMs and VMs to physical resources. The focus is on the VM placement problem which is posed as a multi-objective optimization problem of simultaneously minimizing total resource wastage, power consumption and thermal dissipation costs. An improved genetic algorithm with fuzzy multiobjective evaluation is proposed for efficiently searching the large solution space and conveniently combining possibly conflicting objectives.

A simulation-based evaluation using power-consumption demonstrates the good performance, scalability and robustness. Our approach can seek and find solutions that exhibit good balance among the conflicting objectives while others cannot. [3]

\subsection{Energy efficient vm scheduling for cloud data canters:} Exact allocation and migration algorithms:

For energy, efficient scheduling of virtual machines (VMs) in cloud data centers. Modeling of energy aware allocation and consolidation to minimize overall energy consumption leads us to the combination of an optimal allocation algorithm with a consolidation algorithm relying on migration of VMs at service departures. The optimal allocation problem with a minimum power consumption objective. It is compared with an energy aware best fit algorithm. The exact migration algorithm results from a linear and integer formulation of VM migration to adapt placement when resources are released.

The proposed migration is general and goes beyond the current state of the art by minimizing both the number of migrations needed for consolidation and energy consumption in a single algorithm with a set of valid inequalities and conditions. Experimental results show the benefits of combining the allocation and migration algorithms and demonstrate their ability to achieve significant energy savings while maintaining feasible convergence times when compared with the best fit heuristic. [4]

2.5 Virtual machine resource allocation in cloud computing via multi-agent fuzzy control:

Dynamic resource (re)-allocation for virtual machines in cloud computing is important to guarantee application performance and to reduce operating costs. The problem is to find an adequate trade-off between these two conflicting goals.

Our approach is presented to support the Virtual Machine Monitor in performing resource allocation of VMs running on a physical machine of a cloud provider by expressing the two objectives in a utility function and optimizing this function using fuzzy control.

To potentially work for an increased number of virtual machines, a multiagent fuzzy controller is realized where each agent optimizes its own local utility function. The multi-agent fuzzy controller is empirically compared to a centralized fuzzy controller and an adaptive optimal control approach. Experimental results show. The effectiveness of the multiagent fuzzy controller in finding an adequate trade-off between performance and cost. [5]

2.6 A power efficient genetic algorithm for resource allocation in cloud computing data centers in Cloud Networking:

One of the main challenges in cloud computing is to increase the availability of computational resources, while minimizing system power consumption and operational expenses. This article introduces a power efficient resource allocation algorithm for tasks in cloud computing data centers. The developed approach is based on genetic algorithms which ensure performance and scalability to millions of tasks.

Resource allocation is performed taking into account computational and networking requirements of tasks and optimizes task completion time and data center power consumption. The evaluation results, obtained using a dedicated open source genetic multi-objective framework called jMetal show that the developed approach is able to perform the static allocation of a large number of independent tasks on homogeneous single-core servers within the same data center with a quadratic time complexity. [6]. 


\section{PROPOSED SYSTEM APPROACH}

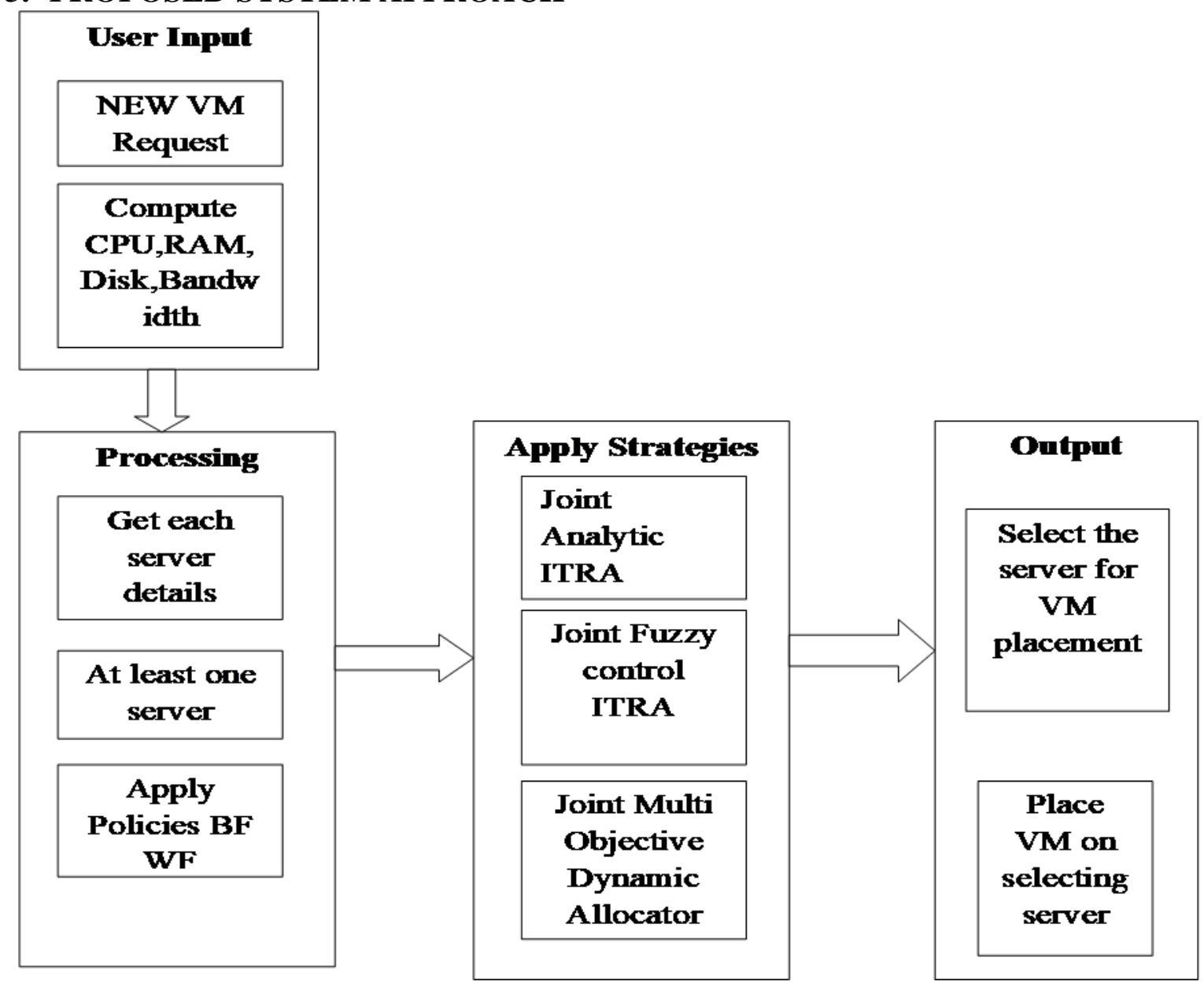

Fig 1 Praposed System

These proposed systems develop to accept as many VM requests as possible; at the same time the network power consumption have to reduce. Each VM request is characterized by four parameters throughput of CPU, RAM, disk and bandwidth.

The server selection includes the following steps:

1) Master node identify server list, i.e., the set of servers with enough IT resources to satisfy the request:

I) Master Node rejected the user request, when list is empty,

ii) Otherwise, go to next step.

2) Master Node has to choose the policy between following: I) Multi Resource Best Fit (BF):

Multi Resource Best Fit selecting the server that has the least resources availability strongly consolidates the system resource utilization;

ii) Multi Resource Worst Fit (WF):

Multi Resource Worst Fit selects the server having the highest resources availability, so as to balance the load among all the available servers.

3) Master Node chooses the best server according to one of the possible following strategies:
I) Analytic ITRA (joint):

Analytic ITRA (A-ITRA) computes for each candidate Server the A-ITRA Availability Index (IA) that takes into Account the availability of IT resources.

II) Fuzzy ITRA (joint):

Fuzzy logic provides a way of dealing with imprecision and nonlinearity in complex control situations. Inputs are passed to an "Interface Engine" where human or experienced-based rules are applied to produce output.

III) Multi-Objective Dynamic Allocator MODA) (joint):

MODA allocates VMs using a technique based on the multiobjective optimization: available resources are the objectives that should be optimized all together.

MODA computes in multiple steps the allocation procedure; When a new VM should be allocated, MODA creates a list of Servers able to fit the request. MODA considers CPUs, RAMs, DISKs and PCs, and it associate the minimum-cost path with each server during the server selection phase. To perform the allocation of VMs:

a) For both computational and network requirements. The joint allocation strategies consider at the same time. 
b) Instead, disjoint strategies split the allocation procedure in two different steps:

i) If server is not available only the computational requirements rejecting the request then select the server where to allocate the VM.

ii) If path is not available then only the bandwidth requirement will be rejecting the request. Then find the minimum-cost path connecting the selected server to the gateway.

4) If server is not available only the computational requirements rejecting the request; Allocation procedure is to choose the server where to allocate the VM evaluating once server get choose then VM get placed.

Proposed System is recommended best strategy for VM allocation approach with simultaneously reduce power consumption, maximize resource utilization and avoid traffic congestion. The outcome of this proposed system is to follow the best strategy for VM allocation considering with minimum power consumption.

\section{CONCLUSION}

In this paper allocator accept as many $\mathrm{VM}$ requests as possible, reducing at the same time the network power consumption. To satisfy the request allocators apply policy between Multi Resource Best Fit (BF) and Multi Resource Worst Fit (WF) to balance the load among all the available servers. To select the best server according to one of the possible strategies disjoint or joint Analytic ITRA, disjoint or joint Fuzzy ITRA, disjoint or joint Multi-Objective Dynamic Allocator MODA) for VM allocation approach with simultaneously reduce power consumption, maximize resource utilization and avoid traffic congestion. The outcome is to follow the best strategy for VM allocation considering with minimum power consumption.

\section{REFERENCES}

[1] C.-T. Yang, K.-C. Wang, H.-Y. Cheng, C.-T. Kuo, and W. C. C. Chu, "Green Power Management with Dynamic Resource Allocation for Cloud Virtual Machines." IEEE, Sep. 2016, pp. 726-733. [Online]. Available: http://ieeexplore.ieee.org/document/6063066/

[2] S. R. M. Amaranth, F. M. Roberto, A. R. Cardoso, and J. Celestino, "Using the Multiple Knapsack Problem to Model the Problem of Virtual Machine Allocation in Cloud Computing." IEEE, Dec. 2015, pp. 476- 483. [Online]. Available: http://ieeexplore.ieee.org/document/6755257/.

[3] J. Xu and J. A. B. Fortes, "Multi-objective virtual machine placement in virtualized data center environments," in Green Computing and Communications (GreenCom), 2016 IEEE/ACM Int'l Conference on Int'l Conference on Cyber, Physical and Social Computing (CPSCom), Dec 2016, pp. 179-188.

[4] C. Ghribi, M. Hadji, and D. Zeghlache, "Energy efficient vm scheduling for cloud data centers: Exact allocation and migration algorithms," in 2016 13th IEEE/ACM International Symposium on Cluster, Cloud, and Grid Computing, May 2016, pp. 671-678.

[5] D. Minarolli and B. Freisleben, "Virtual machine resource allocation in cloud computing via multi-agent fuzzy control," in Cloud and Green Computing (CGC), 2016 Third International Conference on, Sept 2016, pp 188-194.

[6] G. Portaluri, S. Giordano, D. Kliazovich, and B Dorronsoro, "A power efficient genetic algorithm for resource allocation in cloud computing data centers," in Cloud Networking (CloudNet), 2015 IEEE 3rd International Conference on, Oct 2015, pp. 58-63. 